\title{
Within-Host Priority Effects Systematically Alter Pathogen Coexistence
}

\author{
Patrick A. Clay, ${ }^{1, \star}$ Kailash Dhir, ${ }^{2}$ Volker H. W. Rudolf, ${ }^{1}$ and Meghan A. Duffy ${ }^{2}$
}

1. BioSciences Department, Rice University, Houston, Texas 770052; 2. Department of Ecology and Evolutionary Biology, University of Michigan, Ann Arbor, Michigan 48109

Submitted March 2, 2018; Accepted September 8, 2018; Electronically published December 18, 2018

Online enhancements: appendixes. Dryad data: https://dx.doi.org/10.5061/dryad.v118180.

\begin{abstract}
AвSTRACT: Coinfection of host populations alters pathogen prevalence, host mortality, and pathogen evolution. Because pathogens compete for limiting resources, whether multiple pathogens can coexist in a host population can depend on their within-host interactions, which, in turn, can depend on the order in which pathogens infect hosts (within-host priority effects). However, the consequences of within-host priority effects for pathogen coexistence have not been tested. Using laboratory studies with a coinfected zooplankton system, we found that pathogens had increased fitness in coinfected hosts when they were the second pathogen to infect a host, compared to when they were the first pathogen to infect a host. With these results, we parameterized a pathogen coexistence model with priority effects, finding that pathogen coexistence (1) decreased when priority effects increased the fitness of the first pathogen to arrive in coinfected hosts and (2) increased when priority effects increased the fitness of the second pathogen to arrive in coinfected hosts. We also identified the natural conditions under which we expect within-host priority effects to foster coexistence in our system. These outcomes were the result of positive or negative frequency dependence created by feedback loops between pathogen prevalence and infection order in coinfected hosts. This suggests that priority effects can systematically alter conditions for pathogen coexistence in host populations, thereby changing pathogen community structure and potentially altering host mortality and pathogen evolution via emergent processes.
\end{abstract}

Keywords: parasite interactions, coexistence, priority effects, coinfection.

\section{Introduction}

Multiple pathogen strains and species often coexist in host populations (Petney and Andrews 1998; Brogden et al. 2005; Balmer and Tanner 2011; Cox 2011). This has conse-

\footnotetext{
* Corresponding author; email: patrick.clay@rice.edu. ORCIDs: Clay, http://orcid.org/0000-0002-3491-9985; Rudolf, http://orcid .org/0000-0002-9214-2000; Duffy, http://orcid.org/0000-0002-8142-0802.

Am. Nat. 2019. Vol. 193, pp. 000-000. (C) 2018 by The University of Chicago. 0003-0147/2019/19302-58304\$15.00. All rights reserved. DOI: $10.1086 / 701126$
}

quences for host health, as coinfection can increase or decrease host mortality rates and lead to the evolution of higher virulence (Levin and Bull 1994; Lawn 2004; Alizon et al. 2013). However, hosts are a limited resource for which pathogens have to compete, and it is not always possible for multiple pathogens to coexist. One mechanism that can alter pathogen coexistence is how pathogens interact within hosts (Mordecai et al. 2016a). Coexistence is fostered when pathogens facilitate one another, limited when pathogens have negative impacts on one another, and impossible when pathogens prevent coinfection via cross-protection, withinhost resource depletion, or other mechanisms (Gupta et al. 1994; Vasco et al. 2007). These within-host interactions depend strongly on the order in which pathogens infect hosts (Goodman and Ross 1974; Hood 2003; de Roode et al. 2005; Jackson et al. 2006; Jager and Schorring 2006; Lohr et al. 2010; Leung and Poulin 2011; Hoverman et al. 2013; Devevey et al. 2015; Natsopoulou et al. 2015; Sandoval-Aguilar et al. 2015). However, to our knowledge, the impact of within-host priority effects on pathogen coexistence has not been explicitly tested.

Within-host priority effects exist across a range of coinfected taxa and have been demonstrated to alter infection patterns at the host population scale. Within-host priority effects have been found in mammal, plant, amphibian, insect, zooplankton, and fish hosts and emerge from diverse mechanisms. When multiple strains of the same pathogen infect one host, pathogens that arrive first in a host often have a competitive advantage over subsequent strains, possibly due to resource competition or apparent competition through the immune system (Hood 2003; de Roode et al. 2005; Jager and Schorring 2006; Devevey et al. 2015). However, when hosts are coinfected by two (or more) different pathogen species, pathogens may gain an advantage from first or second arrival, depending on the specific system (Goodman and Ross 1974; Al-Naimi et al. 2005; Jackson et al. 2006; Lohr et al. 2010; Leung and Poulin 2011; Hoverman et al. 2013; Natsopoulou et al. 2015; Sandoval-Aguilar 
et al. 2015; Wuerthner et al. 2017). For instance, early arriving pathogens may increase susceptibility in immunecompromised hosts and thereby facilitate infection of laterarriving pathogens (Rolff and Siva-Jothy 2003). Alternatively, vertebrate immune systems are characterized by Th1/Th2 trade-offs (Fenton et al. 2008), which could create positive or negative within-host priority effects between macroparasites and microparasites. Within-host priority effects can scale up to alter epidemic size (Halliday et al. 2017), host population density (Marchetto and Power 2017), average parasite load (Wuerthner et al. 2017), and parasite prevalence (Natsopoulou et al. 2015). However, we still lack a systematic understanding of how different types of within-host priority effects (e.g., late-arriver vs. early arriver benefit) impact coexistence of different pathogen species at the host population level.

We have abundant evidence from free-living communities, however, that priority effects alter community assembly and coexistence. The first organism to arrive in a patch can monopolize resources needed by its competitors, rapidly adapt to local conditions, or increase in size (and thus competitive ability) before competitors arrive (Urban and De Meester 2009; Rasmussen et al. 2014; Fukami 2015). Each of these processes increases the fitness difference between competing community members, reducing coexistence (Chesson 2000; Fukami et al. 2016). The first organism to arrive in a patch may also modify niches available to later-arriving organisms, either increasing or decreasing the fitness of particular community members and thus altering which community members may coexist (Fukami 2015). Priority effects at the patch scale can determine prevalence and coexistence at the metacommunity scale (Urban and De Meester 2009; Tucker and Fukami 2014). Thus, using the framework for priority effects developed in freeliving communities, we expect that priority effects at the host (patch) scale should influence coexistence at the host population (metacommunity) scale.

In addition to gaining insights from the framework for free-living priority effects, our work also builds on earlier theoretical work on how infection order alters coexistence. In single-infection models, the first pathogen to infect a host prevents secondary infections. In this case, only the pathogen with the highest $R_{0}$ will persist within a host population (Gupta et al. 1994; though this may be disrupted by environmental feedbacks: see Lion and Metz 2018). In superinfection models, only one pathogen may infect a host at a time, but secondary pathogens may take over hosts that are first infected by pathogens with a lower virulence (within-host growth rate). In these models, coexistence between pathogens is possible as long as they differ enough in their level of virulence (May and Nowak 1994; Nowak and May 1994). Finally, in coinfection models, multiple pathogens can infect a host simultaneously, and this coexistence at the within-host scale facilitates coexistence at the host population scale as long as pathogen $R_{0}$ from coinfected hosts is greater than 1 (May and Nowak 1995). Further, if the first pathogen to infect a host permanently increases host susceptibility even after the infection is cleared, then pathogen coexistence will expand (Vasco et al. 2007). While these studies take infection order into account, none of them address within-host priority effects in which hosts can be infected with multiple pathogens at once and in which the fitness of both pathogens depends on the order of infection. Given the numerous examples of this type of within-host priority effect (Goodman and Ross 1974; Hood 2003; de Roode et al. 2005; Jackson et al. 2006; Jager and Schorring 2006; Lohr et al. 2010; Leung and Poulin 2011; Hoverman et al. 2013; Devevey et al. 2015; Natsopoulou et al. 2015; Sandoval-Aguilar et al. 2015; Wuerthner et al. 2017), it is important to understand its implications for pathogen coexistence.

Here we suggest that within-host priority effects may systematically alter pathogen coexistence by creating positive or negative frequency dependence. As a pathogen's prevalence increases, so does its probability of being the first pathogen to infect a host. Thus, if a pathogen receives a fitness benefit from first arrival, then its average per capita transmission rate from coinfected hosts should increase along with its prevalence (relative to the coinfecting pathogen's prevalence). This creates positive frequency dependence that should decrease the probability of pathogen coexistence. On the other hand, if a pathogen receives a benefit from second arrival, then its per capita transmission rate from coinfected hosts should increase when its prevalence decreases. This scenario can create negative frequency dependence that should increase coexistence by increasing pathogen fitness when rare.

To explore how within-host priority effects alter pathogen coexistence at the host population level, we measured within-host priority effects in zooplankton coinfected with bacterial and fungal pathogens and then used these measurements to parameterize a pathogen coexistence model that we used to explore the conditions under which within-host priority effects might foster pathogen coexistence or competitive exclusion. Using this system, we specifically asked the following questions: (1) How does the infection order of pathogens impact pathogen and host fitness in our system? (2) How do fitness advantages from first or second arrival alter pathogen coexistence? (3) Under what conditions do we expect within-host priority effects to make the difference between single and multipathogen persistence in our system? We found that within-host priority effects foster pathogen coexistence in our system by creating negative frequency dependence. This suggests that within-host priority effects can be important drivers of multipathogen communities in natural host populations. 


\section{Experimental Methods}

\section{Study System}

Our focal host, Daphnia dentifera, is a cyclically parthenogenetic grazing zooplankton common in stratified lakes in the Midwestern United States (Tessier and Woodruff 2002). While filtering, $D$. dentifera incidentally ingests the two pathogens used in our study, the bacterium Pasteuria ramosa and the fungus Metschnikowia bicuspidata. Both pathogens are obligate killers. After being ingested, they replicate within $D$. dentifera until host death, at which point they are released into the water column once more until being ingested by a new host. The two pathogens differ in how they reduce host fitness. Metschnikowia replicates quickly and reduces host life span, while Pasteuria castrates hosts (Auld et al. 2012, 2014).

\section{Experimental Setup}

We used a clonal line of D. dentifera (Mid37) that was originally collected from Midland Lake, Indiana, and that has been maintained asexually in lab conditions for several years. In order to standardize effects of the environment, we reared individuals singly in beakers and isolated individuals from the third clutch. We then isolated individuals from the third clutch of this second generation of animals and used those individuals in our experiment. Throughout the experiment, hosts were kept singly in separate individual beakers filled with $30 \mathrm{~mL}$ of filtered lake water. For both pathogens, we used an isolate that had been grown in the lab for several years (Standard for Metschnikowia and G/18 for Pasteuria). This host clone and these pathogen isolates have been used successfully in previous work to study host-pathogen interactions (Auld et al. 2012, 2014).

We tested for within-host priority effects by exposing hosts to either Pasteuria or Metschnikowia, to both pathogens in different arrival orders, or to a control treatment with no pathogens, resulting in eight treatments (table 1). Each treatment was replicated with 30 individuals that were maintained singly in a beaker filled with $30 \mathrm{~mL}$ of filtered lake water. On day 7, treatments 2-6 (table 1) were exposed to either 1,000 spores/mL of Pasteuria and/or 500 spores/ $\mathrm{mL}$ of Metschnikowia. Spore doses were chosen in an attempt to yield similar, high levels of infection, based on prior studies (M. A. Duffy, unpublished data). After 48 h, all individuals were placed in new water. On day 12 , treatments 5-8 were exposed to either 1,000 spores/mL of Pasteuria or 500 spores/mL of Metschnikowia. Again, after 48 h, all individuals were placed in new water. The experiment ended on day 47, at which point all but four hosts had died.

\section{Response Variables}

Host Fitness. Host fitness was measured in terms of life span and number of offspring per day. Three times each week, we assessed whether individuals had died and counted the number of offspring produced per host. During inspection, hosts were transferred to clean beakers; offspring were not transferred.

Pathogen Fitness. We examined how within-host priority effects altered the number of infectious propagules created by an infection, or spore yield, which is a proxy for pathogen fitness. In our system, infectious spores are only released into the environment from a host after death. Thus, secondary infections are proportional to the number of spores found in a host at death. Dead hosts were preserved in $100 \mu \mathrm{L}$ of nano-pure water. Preserved hosts were then ground up, and total Pasteuria and Metschnikowia spores per host were recorded.

\section{Data Analysis}

We tested for specific pairwise differences in spore yield among treatments to address three questions. First, we asked whether coinfection altered spore yield. We thus compared our single-infection treatments to our coinfection treatments, which shared pathogen infection timing ( 2 vs. 4,2 vs. 5 , and 6 vs. 7 for Pasteuria and 3 vs. 4,3 vs. 6 , and 5 vs. 8 for Metschnikowia). Second, we asked

Table 1: Experimental treatments

\begin{tabular}{llll}
\hline Treatment no. & \multicolumn{1}{c}{ Treatment name } & Day 7 & Day 12 \\
\hline 1 & Uninfected & None & None \\
2 & Early Pasteuria & Pasteuria & None \\
3 & Early Metschnikowia & Metschnikowia & None \\
4 & Simultaneous infection & Pasteuria and Metschnikowia & None \\
5 & Pasteuria first & Pasteuria & Metschnikowia \\
6 & Metschnikowia first & Metschnikowia & Pasteuria \\
7 & Late Pasteuria & None & Pasteuria \\
8 & Late Metschnikowia & None & Metschnikowia \\
\hline
\end{tabular}

Note: Hosts were exposed to Pasteuria at 1,000 spores/mL and Metschnikowia at 500 spores/mL on days 7 and 12 . 
whether infection order altered spore yield from coinfected hosts, comparing our sequential coinfection treatments (5 vs. 6). Third, we asked whether differences in spore yield due to arrival order in coinfected hosts could be explained by differences in when each pathogen arrived. Thus, we compared our single-infection treatments where pathogens arrived on day 7 versus 12 (2 vs. 7 for Pasteuria and 3 vs. 8 for Metschnikowia). We calculated significance of pairwise differences using the glht function in the multcomp package in $\mathrm{R}$, correcting for the false discovery rate due to multiple comparisons (Benjamini and Hochberg 1995). All response variables had a high level of heteroscedasticity, so variance was allowed to vary across treatments using the gls function in the nlme4 package in R. We used the same methods to test pairwise differences between life span and offspring, though for these variables, we tested all pairwise comparisons.

Four individuals lived till the end of the experiment (one in treatment 1 , one in treatment 2 , and two in treatment 6). These individuals were not included in calculations of host life spans. Including these individuals in our analysis assuming slightly extended life spans ( 2 days longer than the experiment) does not change statistical results. Individuals were only included in analyses if they were successfully infected by all pathogens to which they were exposed. Pasteuria spores were found in four individuals that were not exposed to Pasteuria (three in treatment 3 and one in treatment 8). These individuals were removed from all analyses. In addition, hosts that had partially decomposed between death and collection were not included in mean spore counts (22 out of 240 hosts), as hosts begin to lose spores with decomposition.

\section{Experimental Results}

In our figures, we show only results from treatments that were used to parameterize our coexistence model. Full results including all treatments can be found in the appendix (figs. B1-B4; figs. B1-B9 are available online).

\section{Pathogen Fitness}

We found that within-host priority effects determined Metschnikowia spore yield, whereas Pasteuria spore yield was only determined by whether it was in a singly infected or coinfected host. We found that coinfection significantly reduced Pasteuria spore yield when comparing treatments 2 and 5 (early Pasteuria vs. Pasteuria first; $p=.022$; "single" vs. "first" in the top panel of fig. 1) and 6 and 7 (Metschnikowia first vs. late Pasteuria; $p=.0016$ ). Within coinfections, arrival order did not significantly impact Pasteuria spore yield ( $p=.93$ for comparing sequential coinfection treatments; "first" vs. "second" in the top panel

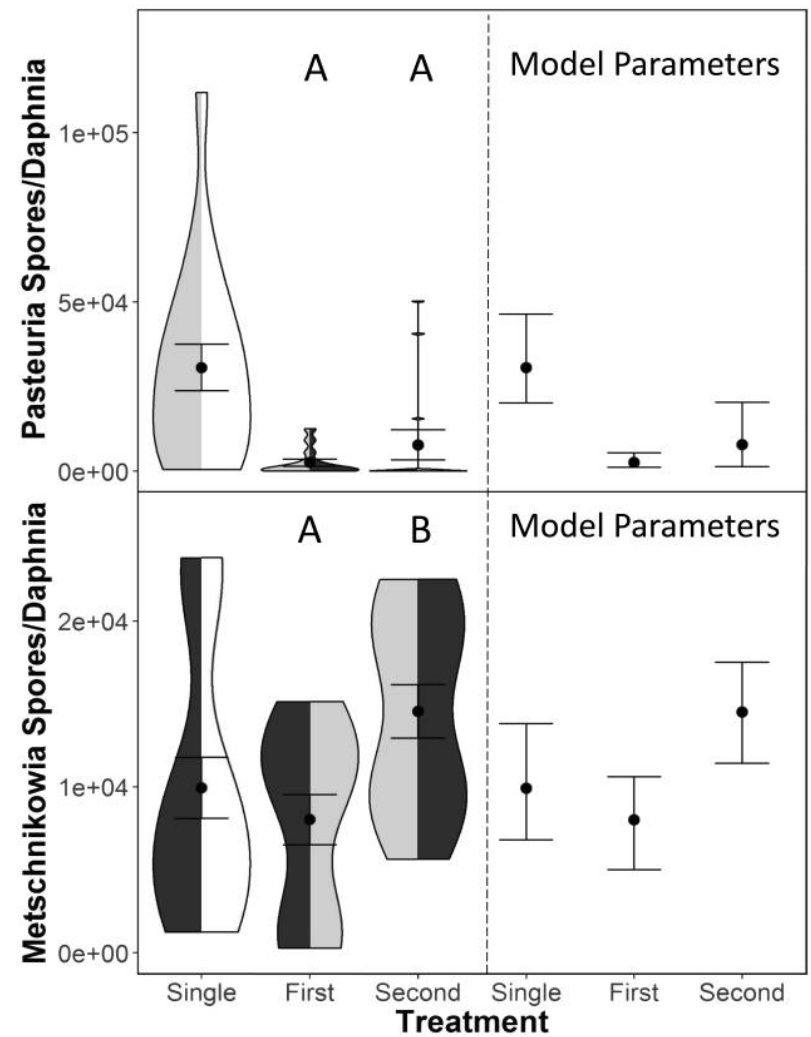

Figure 1: Number of spores found in hosts infected with Pasteuria (top) and Metschnikowia (bottom). Left, violin plots show the distribution of spore yield from singly infected hosts ("single"), coinfected hosts infected first by the focal pathogen ("first"; the focal pathogen is Pasteuria on the top and Metschnikowia on the bottom), and coinfected hosts infected second by the focal pathogen ("second"), with points representing average spore yield and bars representing standard error. The color of the left half of each violin plot indicates the infection treatment on day 7 (white $=$ no pathogen, light gray $=$ Pasteuria, dark gray $=$ Metschnikowia), while the color on the right half of each violin plot indicates the infection treatment on day 12. Spore yield from sequential coinfection treatments with different letters were significantly different, indicating the presence of priority effects. Right, shown are bootstrapped means and 95\% confidence intervals of spore yield used to parameterize the coexistence model. Plots including individual data points from all treatments are included in the appendix (figs. B1, B2). Data underlying all figures are deposited in the Dryad Digital Repository: https:// dx.doi.org/10.5061/dryad.v118180 (Clay et al. 2019).

of fig. 1). Metschnikowia, on the other hand, had a significantly higher spore yield from coinfected hosts in which it arrived second than from coinfected hosts in which it arrived first ( $p=.042$; "first" vs. "second" in the bottom panel of fig. 1). However, spore yield from neither coinfection treatment was significantly different than that from singly infected hosts when comparing treatments 3 and 
6 (early Metschnikowia vs. Metschnikowia first; $p=.51$; "single" vs. "first" in the bottom panel of fig. 1) or 5 and 8 (Pasteuria first vs. late Metschnikowia; $p=.74$ ). Thus, Pasteuria spore yield was determined by whether the host was coinfected and displayed no significant within-host priority effects, whereas Metschnikowia spore yield was driven by within-host priority effects in coinfections more so than by whether the host was coinfected.

\section{Host Fitness}

The key driver of host life span was Metschnikowia infection: Daphnia that were infected with Metschnikowia (treatments 3,5, and 6) lived on average $13 \pm 1.1$ days after day 7 (day of first possible infection), whereas Daphnia that were not exposed to Metschnikowia (treatments 1 and 2) lived on average $27 \pm 1.0$ days after day 7 (fig. 2; error estimates are standard error of the mean). Based on pairwise comparisons corrected for false discovery rate, there was a significant difference in life span $(p<.001$ for all comparisons) between all Metschnikowia-infected and Metschnikowiauninfected treatments. There was no significant difference in life span in pairwise comparisons among treatments infected by Metschnikowia, nor was there a significant difference in pairwise comparisons among treatments that did not include Metschnikowia. Thus, host life span was deter- mined by whether individuals were infected by Metschnikowia, while Pasteuria infection had no significant effect on host life span.

All infections reduced host fecundity (fig. 3). Pairwise comparisons corrected for false discovery rate showed that unexposed hosts had significantly greater fecundity than all infected hosts $(p<.001$ for all comparisons). There were no significant differences in fecundity between Metschnikowia infected, Pasteuria infected, and coinfected hosts. Uninfected hosts had, on average, $4.1 \pm 0.14$ offspring per day, while infected hosts had $1.6 \pm 0.16$ offspring per day.

\section{Model Methods \\ Model Equations}

To test for the conditions under which we find coexistence in our system, we used a susceptible-infected model with environmental transmission to model a Daphnia population infected by Pasteuria and Metschnikowia with spore densities $P$ and $M$, respectively. Hosts can be susceptible $(S)$, singly infected by either pathogen $\left(I_{P}, I_{M}\right)$, or coinfected. To keep track of within-host priority effects, coinfected hosts are divided into two groups: those where Pasteuria arrived first $\left(C_{P M}\right)$ and those where Metschnikowia

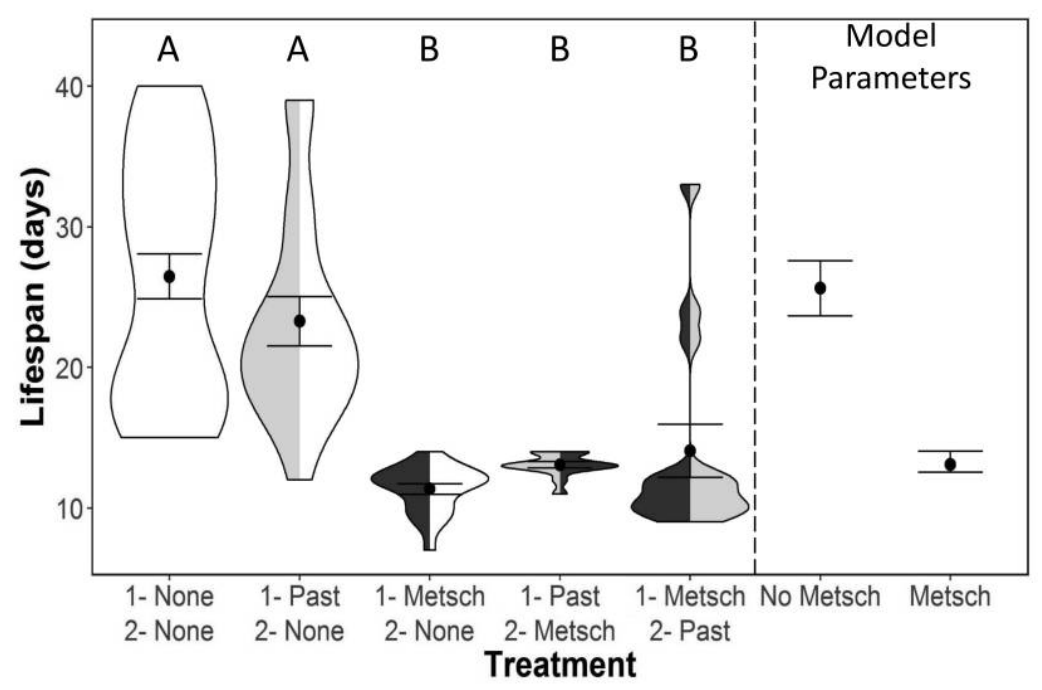

Figure 2: Number of days past initial infection date (day 7) that individuals survived. Left, violin plots show the distribution of life spans within each treatment, with points representing average life span and bars representing standard error. The color of the left half of each violin plot indicates the infection treatment on day 7 (white $=$ no pathogen, light gray $=$ Pasteuria, dark gray $=$ Metschnikowia, also indicated in the top row of the $X$-axis), while the color on the right half of each bar plot indicates the infection treatment on day 12 (also indicated in the bottom row of the $X$-axis). Treatments with shared letters were not significantly different. Right, shown are bootstrapped means and $95 \%$ confidence intervals of postinfection life spans of individuals uninfected and infected by Metschnikowia used to parameterize the coexistence model. A plot including individual data points from all treatments (including the simultaneous coinfection treatment) is included in the appendix (fig. B3). 


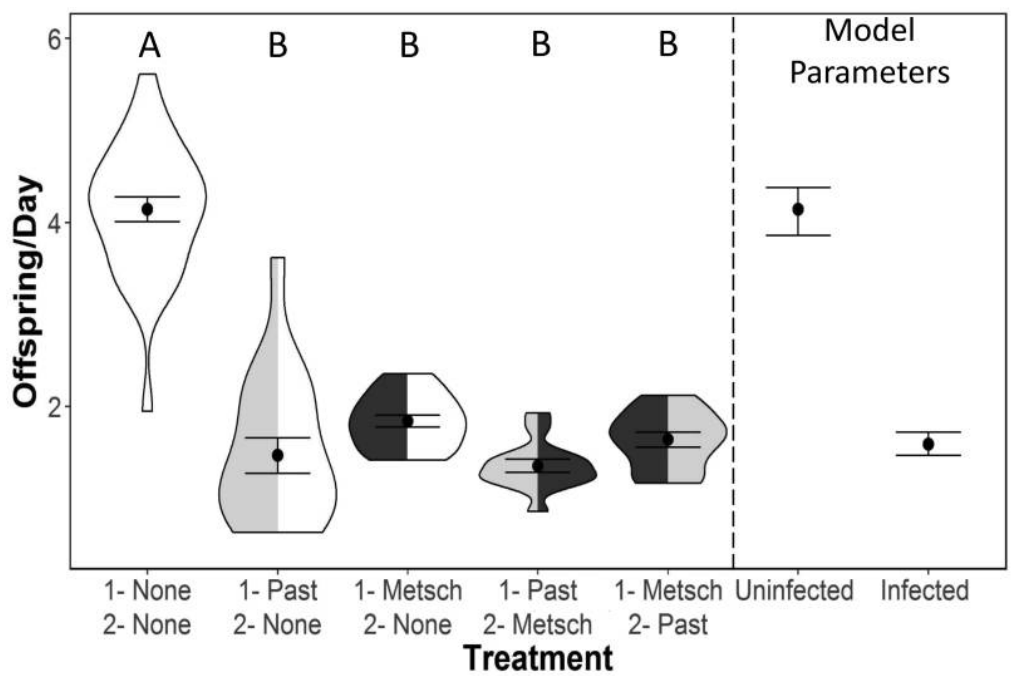

Figure 3: Average offspring per day. Left, violin plots show the distribution of offspring/day within each treatment, with points representing average offspring/day and bars representing standard error. The color of the left half of each violin plot indicates the infection treatment on day 7 ( white $=$ no pathogen, light gray $=$ Pasteuria , dark gray $=$ Metschnikowia, also indicated in the top row of the $X$-axis), while the color on the right half of each bar plot indicates the infection treatment on day 12 (also indicated in the bottom row of the $X$-axis). Treatments with shared letters were not significantly different. Right, shown are bootstrapped means and $95 \%$ confidence intervals of offspring/day of uninfected and infected individuals used to parameterize the coexistence model. A plot including individual data points and all treatments is included in the appendix (fig. B4)

arrived first $\left(C_{M P}\right)$. We modeled chronic infections with no recovery as hosts cannot clear infections in our system. We initially incorporated a simultaneously coinfected class in our model. However, when we derived an analogous discrete time model for this system, with each time step representing 1 day, the proportion of coinfected hosts that were infected by both pathogens in a single day was $<0.01$ for all parameter values explored, so we removed the simultaneous coinfection class for simplicity.

Susceptible hosts are born into the population via all infection classes (no vertical transmission) and become singly infected by consuming spores in the environment, $\left(f \mu_{P} P+f \mu_{M} M\right) S$. Dynamics of the susceptible host class are given by

$$
\begin{aligned}
\frac{d S}{d t}= & \overbrace{b\left(S, I_{P}, I_{M}, C_{P M}, C_{M P}\right)\left(1-\frac{N}{K}\right)}^{\text {births }} \\
& -\overbrace{\left(f \mu_{P} P+f \mu_{M} M\right) S}^{\text {infection }}-\overbrace{d_{S} S}^{\text {deaths }},
\end{aligned}
$$

where $\mu_{P}$ and $\mu_{M}$ are the per spore infectivities of Pasteuria and Metschnikowia, respectively; $f$ is the host feeding rate; $K$ is host carrying capacity; $N$ is total population size; $b(S$, $\left.I_{P}, I_{M}, C_{P M}, C_{M P}\right)$ is the birthrate; and $d_{S}$ is the death rate. Here $b\left(S, I_{P}, I_{M}, C_{P M}, C_{M P}\right)$ is the summed birthrate of each infection class, given by

$$
\begin{gathered}
b\left(S, I_{P}, I_{M}, C_{P M}, C_{M P}\right) \\
=\overbrace{b_{S} S}^{\text {offspring from } S}+\overbrace{b_{I}\left(I_{P}+I_{M}+C_{P M}+C_{M P}\right)}^{\text {offspring from infected hosts }} .
\end{gathered}
$$

Since most infected individuals have similarly decreased fecundity compared to uninfected individuals (fig. 3), in fected hosts have a birthrate $b_{I}$ and susceptible hosts have a birthrate $b_{s}$.

Singly infected hosts can transition to the coinfected host class by consuming spores of the pathogen they are not infected by $\left(f \mu_{M} I_{P} M\right.$ or $\left.f \mu_{P} I_{M} P\right)$. Since Metschnikowia infection reduces host life span (fig. 2), all hosts infected by Metschnikowia die as a function of Metschnikowiainduced mortality $\left(d_{M}\right)$. Changes in the numbers of infected and coinfected hosts are then given by

$$
\begin{aligned}
\frac{d I_{P}}{d t} & =\overbrace{f \mu_{P} S P}^{\text {infection }}-\overbrace{f \mu_{M} I_{P} M}^{\text {coinfection }}-\overbrace{d_{S} I_{P}}^{\text {deaths }}, \\
\frac{d I_{M}}{d t} & =\overbrace{f \mu_{M} S M}^{\text {infection }}-\overbrace{f \mu_{P} I_{M} P}^{\text {coinfection }}-\overbrace{d_{M} I_{M}}^{\text {deaths }}, \\
\frac{d C_{P M}}{d t} & =\overbrace{f \mu_{M} I_{P} M}^{\text {coinfection }}-\overbrace{d_{M} C_{P M}}^{\text {deaths }},
\end{aligned}
$$




$$
\frac{d C_{M P}}{d t}=\overbrace{f \mu_{P} I_{M} P}^{\text {coinfection }}-\overbrace{d_{M} C_{M P}}^{\text {deaths }} .
$$

Increasing host population density does not increase host death rate in our model, as this would decrease the available growth time - and thus spore yield - for each pathogen. As we did not measure this relationship, we did not include it in our model, but see Auld et al. (2014) for discussion of how earlier mortality of infected hosts could alter spore yield - and thus host persistence - in this system.

Infected hosts transmit spores into environmental spore pools, whose dynamics are given by

$$
\frac{d P}{d t}=\overbrace{d_{S} \beta_{P(P)} I_{P}+d_{M} \beta_{P(P M)} C_{P M}+d_{M} \beta_{P(M P)} C_{M P}}^{\text {spore release }}-\overbrace{\alpha_{P} P}^{\text {loss }},
$$

$$
\begin{aligned}
\frac{d M}{d t}= & \overbrace{d_{M} \beta_{M(M)} I_{M}+d_{M} \beta_{M(P M)} C_{P M}+d_{M} \beta_{M(M P)} C_{M P}}^{\text {spore release }} \\
& -\overbrace{\alpha_{M} M}^{\text {loss }}-\overbrace{f M N}^{\text {uptake }},
\end{aligned}
$$

where $\beta_{i(j)}$ represents the number of spores $i$ from host class $j$, corresponding to spore yields in figure 1. Thus, all hosts that are infected by a given pathogen add spores of that pathogen to the environment on death. Spores have a loss rate $\left(\alpha_{i}\right)$, which represents spore degradation and spores moving out of the system (e.g., due to settling). Metschnikowia spores are removed from the environment by host feeding $(f)$, an important driver of disease dynamics in this system (Civitello et al. 2013). Pasteuria spores, alternatively, can survive passage through the host gut and thus are not removed by host feeding (King et al. 2013).

\section{Model Parameterization}

To parameterize our model, we used bootstrapping methods with 5,000 replicate draws from the boot and boot.ci functions in $\mathrm{R}$ to estimate the mean and $95 \%$ confidence intervals of spore yield, host fecundity, and host life span from our empirical results. Treatments were grouped for this analysis as described above and shown in figures 1-3. Derivation of host birthrate, death rate, and all other parameters are described in appendix B (apps. A, B are available online), along with a table of all parameter values (table A1; tables A1, B1 are available online). Our model was deterministic and used single mean parameter values for our main results. However, we additionally ran our model across the parameter space given by the $95 \%$ confidence intervals of our parameters to check model sensitivity.

\section{Incorporating Priority Effects}

We ran our model under three scenarios to compare coexistence predictions: (1) pathogens maximize their fitness in coinfected hosts by being the first pathogen to arrive (first-arrival advantage scenario), (2) arrival order does not alter pathogen fitness (no-advantage scenario), and (3) pathogens maximize their fitness in coinfected hosts by being the second pathogen to arrive (second-arrival advantage scenario). Both pathogens had higher spore yield from coinfected hosts when they arrived second (though this was only significant for Metschnikowia; fig. 1). Thus, for our second-arrival advantage scenario, we set spore yield from coinfected hosts equal to that found in our experiments. For our first-arrival advantage scenario, we switched the spore yields of both pathogens from coinfected hosts where Pasteuria arrived first and coinfected hosts where Metschnikowia arrived first. For our no-advantage scenario, we averaged the spore yields of both pathogens across both sequentially infected host classes by pooling the spore yields from both our sequential infection treatments and bootstrapping new mean spore yield estimates. Exact parameter values can be found in the appendix (table A1).

\section{Testing Coexistence}

To test coexistence of Pasteuria and Metschnikowia, we initialized our model with a susceptible Daphnia population at carrying capacity with one individual in each singly infected class and recorded whether neither, one, or both pathogens were circulating in the host population at equilibrium conditions. We ran our model using the lsoda function in the deSolve package in $\mathrm{R}$. To test whether initial conditions altered equilibrium values, we independently varied the initial prevalence of each pathogen from $1 \%$ to $100 \%$. For the ranges of parameter values we considered, we found that stable states were not dependent on initial prevalence. If the sum of all infection classes for a pathogen was reduced to less than 0.01 , that pathogen was considered to be extinct. We also conducted a formal invasion analysis, which confirmed our numerical results (fig. B7).

We tested how pathogen coexistence varied along a fitness gradient of both hosts by varying spore loss rates of both pathogens $\left(\alpha_{P}\right.$ and $\left.\alpha_{M}\right)$. Decreasing the spore loss rate of a pathogen increases pathogen $R_{0}$. Varying the spore loss rate of pathogens is a good proxy for how pathogen fitness changes over space and time in natural settings, as there is evidence that environmental variables influence spore loss (Overholt et al. 2012). We examined pathogen coexistence for spore loss rates ranging from approximately one-seventieth to 1.9 times (for Metschnikowia) and one- 
ninetieth to 1.6 times (for Pasteuria) the loss rates measured under laboratory conditions (app. A).

\section{Model-Coexistence Results}

The Impact of First-versus Second-Arrival Advantage on Coexistence

Coexistence of both pathogen species in the host population varied depending on the presence and type of priority effect. Compared to systems without within-host priority effects, pathogens were more likely to coexist in a host population if they had second-arrival advantage but less likely to coexist if they had first-arrival advantage (fig. 4). The parameter space in which Pasteuria could exist was not sensitive to whether pathogens gained an advantage from first or second arrival. Instead, the likelihood of coexistence was driven by the parameter space in which Metschnikowia could maintain itself in a host population. Metschnikowia had a much larger parameter space in which it could exist with second-arrival advantage than with first-arrival advantage.

These coexistence patterns can be explained by $(a)$ the prevalence of coinfections and $(b)$ the frequency dependence created by within-host priority effects. As we decrease the spore loss rate of a pathogen (and thus increase its fitness), the prevalence of that pathogen increases. Further, as the prevalence of one pathogen increases, more of the infections by the other pathogen are in coinfected hosts. For example, as we decrease Metschnikowia's loss rate, the proportion of hosts infected by Pasteuria that are also coinfected by Metschnikowia increases. Pasteuria spore yield is significantly reduced by coinfection (fig. 1). Thus, decreasing Metschnikowia spore loss also decreases the average spore yield of Pasteuria (fig. 5A-5C). On the other hand, Metschnikowia's average spore yields in sequentially coinfected hosts and in singly infected hosts are approximately the same (fig. 1). However, Metschnikowia spore yield is dependent on the order in which pathogens arrive in coinfected hosts. As we decrease Metschnikowia prevalence, Metschnikowia is more likely to arrive second in coinfected hosts. If Metschnikowia spore yield was decreased when arriving second in coinfected hosts, then it would have low per capita spore yield at low prevalence, preventing coexistence (fig. 5D). However, since Metschnikowia has an increase in spore yield from arriving second in coinfected hosts (fig. 4), it will have high per capita spore yield at low prevalence (fig. $5 F$ ). This creates negative frequency dependence, which facilitates coexistence.

\section{Conditions under Which Within-Host Priority Effects Determine Coexistence}

We expect within-host priority effects to foster coexistence between Metschnikowia and Pasteuria when the Metschnikowia loss rate is high. Under loss rates found in lab conditions ( $\alpha_{P}=0.31, \alpha_{M}=1.2$; see app. B for estimation), our model indicates pathogen coexistence regardless of within-host priority effects (fig. 6A). However, in field conditions, we expect loss to be higher as infectious spores are exposed to ultraviolet light, extreme temperatures, and settling out of the water column (Overholt et al. 2012; Civitello et al. 2013; Shocket et al. 2018). Thus, we examined whether

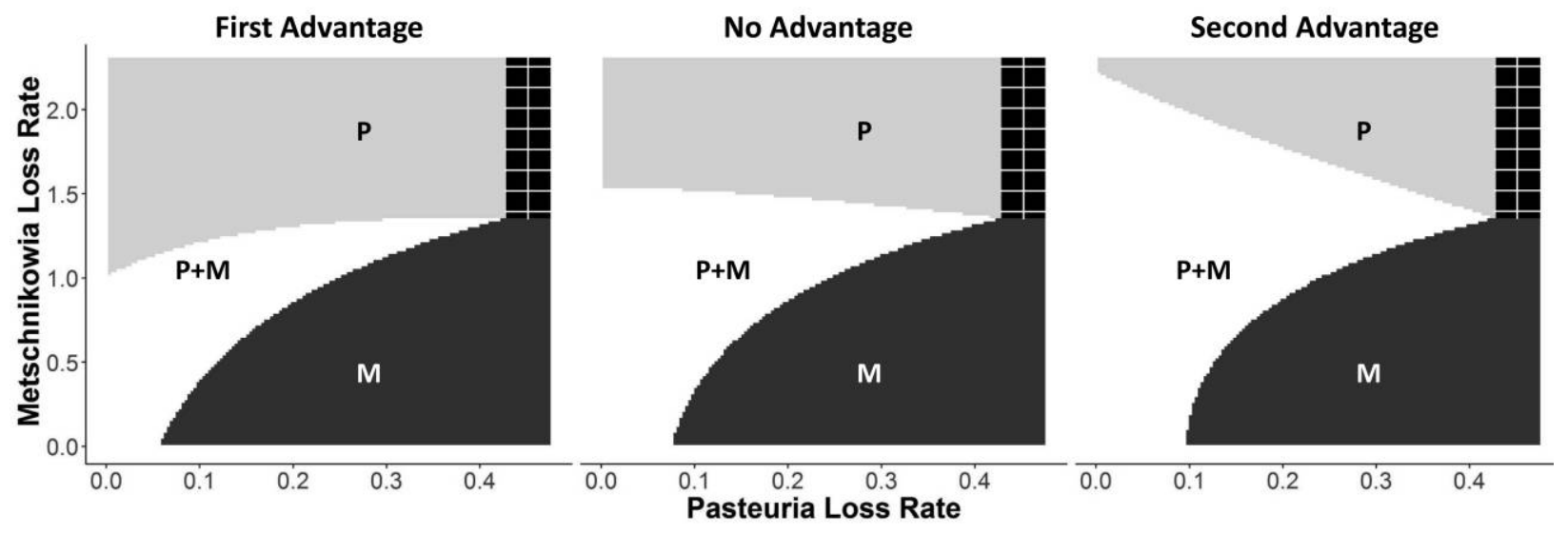

Figure 4: Coexistence phase planes of Metschnikowia and Pasteuria in a shared population across a gradient of spore loss rates of Metschnikowia ( $X$-axis) and Pasteuria ( $Y$-axis): when the first pathogen to arrive has a fitness advantage, when the arrival order does not matter, and when the second pathogen to arrive has a fitness advantage. Pathogen fitness increases along both axes. Plots show whether only Pasteuria (light gray), only Metschnikowia (dark gray), both pathogens (white), or neither pathogen (black with grid marks) can maintain themselves across a parameter space of Metschnikowia and Pasteuria loss rates. 


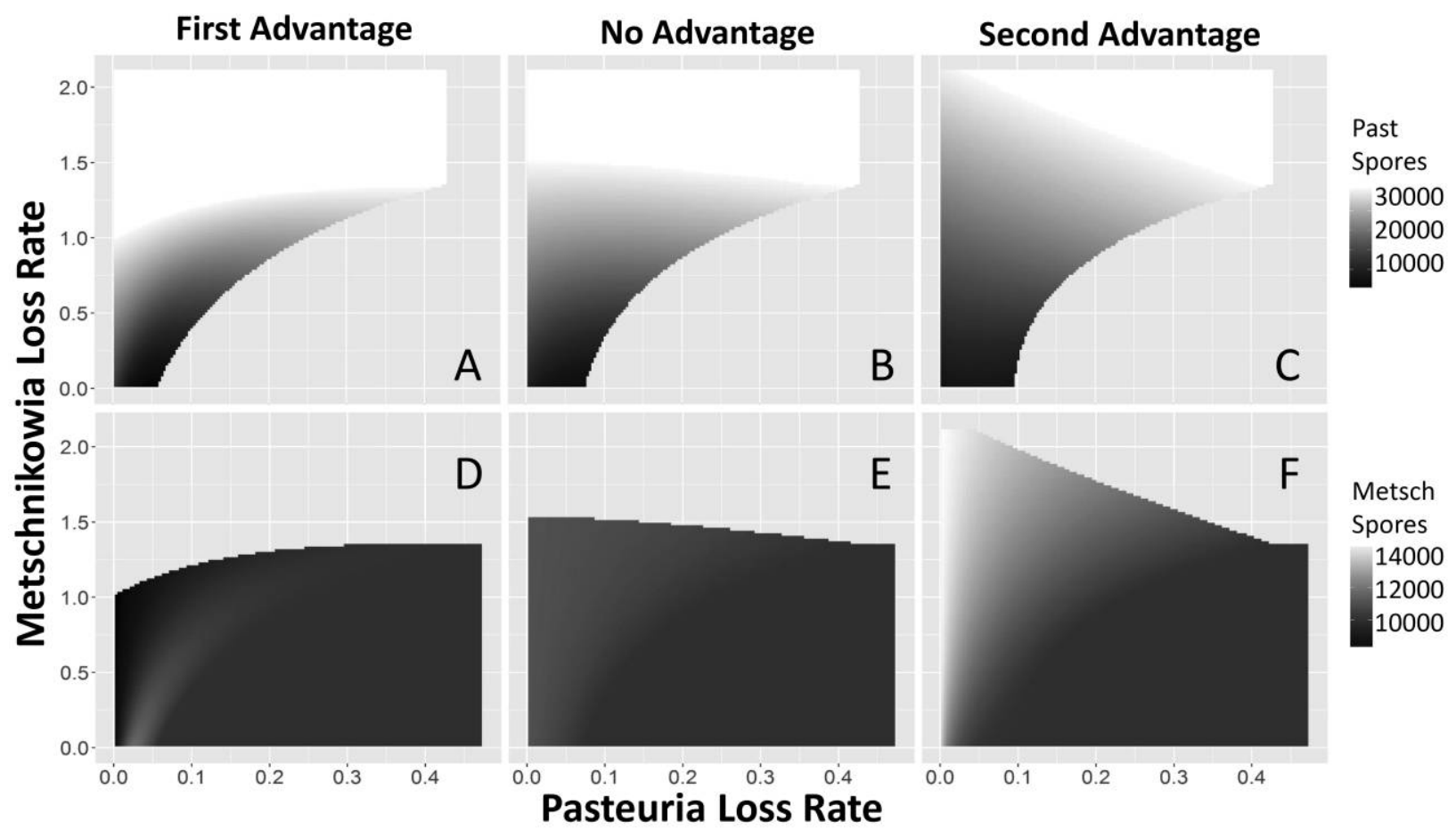

Figure 5: Mean spore yield of Pasteuria $(A-C)$ and Metschnikowia $(D-F)$ from infected hosts when pathogens have first-arrival advantage $(A, D)$, no arrival advantage $(B, E)$, and second-arrival advantage $(C, F)$. Scale bars represent the spore yield gradient for each row, from black representing low spore yield to white representing high spore yield. Gridded parts of the phase plane show the parameter space in which the given pathogen cannot maintain itself within the host population.

within-host priority effects foster coexistence if we increased Metschnikowia loss rate, Pasteuria loss rate, or the loss rates of both. Note that it is biologically reasonable to independently vary Pasteuria and Metschnikowia loss rates because the pathogens respond differently to environmental variables; for example, Metschnikowia is more sensitive to ambient radiation in lakes than Pasteuria (C. L. Shaw, S. R. Hall, E. P. Overholt, C. E. Cáceres, C. E. Williamson, and M. A. Duffy, unpublished data). We increased the Metschnikowia loss rate to values that approximate conditions where $78 \%$ of all Metschnikowia spores are lost from the water column each day $\left(\alpha_{M}=1.5\right)$ and increased the Pasteuria loss rate to values that approximate conditions where $28 \%$ of all Pasteuria spores are lost from the water column each day $\left(\alpha_{P}=0.33\right)$. We found that with a high Metschnikowia loss rate, within-host priority effects (second-arrival advantage in accordance with our empirical results) allow for both pathogens to coexist, whereas only Pasteuria may persist if arrival order does not alter pathogen spore yield (fig. 6B). Increasing the Pasteuria loss rate as well provides a similar pattern, though it is less likely that within-host priority effects will create coexistence (fig. 6C). Finally, if we only increase the Pasteuria loss rate, only Metschnikowia may persist regardless of priority effects. When Met- schnikowia loss rates were high, within-host priority effects that gave advantage to first arrivers limit pathogen coexistence (fig. 6).

\section{Discussion}

In natural systems, hosts can harbor a diverse community of pathogen species, but it is still unclear what factors structure the composition of these pathogen communities. Our study shows that within-host priority effects influence pathogen fitness and scale up to alter pathogen coexistence patterns in host populations. Specifically, we found that infection order played an important role in determining spore yield (and, hence, transmission) of the fungal pathogen Metschnikowia: when Metschnikowia arrived second, it had a higher spore yield than when it arrived first in a coinfected host. This benefit of arriving second within a coinfected individual scales up to influence populationlevel patterns, increasing the likelihood of pathogen coexistence in our system. In addition, we found that if Metschnikowia has a higher spore loss rate than coinfecting pathogens (as we have previously measured in natural environments), within-host priority effects should alter coexistence outcomes. These results demonstrate that with- 


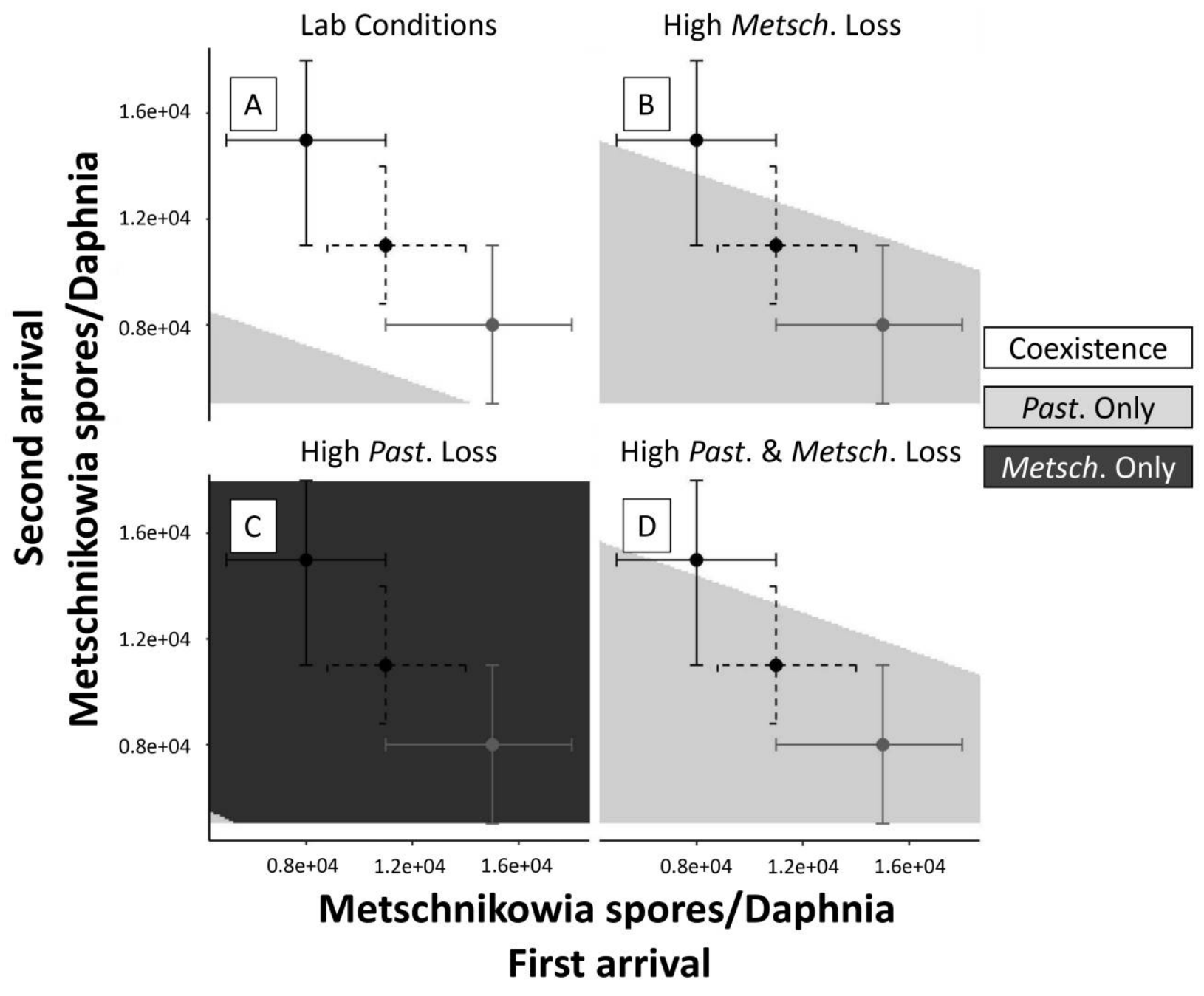

Figure 6: Within-host priority effects are most likely to drive coexistence in scenarios with high Metschnikowia spore loss rates. The axes represent the number of Metschnikowia spores released from coinfected hosts in which Metschnikowia is the first to arrive (X-axis) or second to arrive ( $Y$-axis). Points surrounded by black, solid bars correspond to bootstrapped estimates of the mean and $95 \%$ confidence intervals found for our empirical spore yield data (fig. 3). This is our scenario for within-host priority effects. For points surrounded by dashed bars, we repeated the same bootstrapping procedure but lumped together the Metschnikowia spore yields from all hosts that were coinfected sequentially. Thus, this is our scenario for no within-host priority effects, where the number of spores released from hosts where Metschnikowia arrives first is the same as from hosts where it arrives second. Points surrounded by gray confidence intervals represent spore yield if pathogens gain first-arrival advantage. We did not focus on Pasteuria spore yield because altering the order of pathogen arrival did not alter Pasteuria spore yield.

in-host priority effects can have systematic impacts on disease patterns at the host population scale in natural populations, potentially affecting host mortality, pathogen evolution, and pathogen prevalence (Abu-Raddad et al. 2006; Alizon et al. 2013).

When multiple pathogens can infect the same host, they also compete for resources, both at the within-host and between-host scale, which can lead to competitive exclusion of pathogens in host populations. Within hosts, pathogens may compete for resources such as nutrients, may directly interfere with one another, or may trigger apparent competition via the immune system (Gardner et al. 2004; Pedersen and Fenton 2007; Wale et al. 2017). At the between-host scale, pathogens compete for susceptible hosts. In the absence of coexistence mechanisms, pathogens that compete for these limiting resources cannot coexist, and barring complex environmental feedbacks, only the pathogen with the highest fitness $\left(R_{0}\right)$ will persist in a host population (Gupta et al. 1994; Lion and Metz 2018). Stabilizing coexistence mechanisms can promote coexis- 
tence of competing pathogens by increasing the fitness of an organism when it is rare (Chesson 2000). This may be achieved via niche differentiation, where pathogens use different within-host resources, age classes of hosts, or areas within the host's body (Power 1996; Fitt et al. 2006). Additional stabilizing mechanisms include competition colonization trade-offs and specialization to different vectors (Ojosnegros et al. 2010; Mordecai et al. 2016a, 2016b). Such stabilizing mechanisms can help explain the rich pathogen and parasite communities that have been observed in an abundance of host populations (Petney and Andrews 1998; Brogden et al. 2005; Cox 2011).

Our work expands the current framework of pathogen coexistence by demonstrating that feedbacks between pathogen prevalence and within-host pathogen interactions can have stabilizing or destabilizing impacts on pathogen coexistence. Within-host priority effects that give advantage to the first pathogen to infect a host can decrease the likelihood of pathogen coexistence by decreasing the fitness of pathogens at low prevalence (figs. 4, 5). On the other hand, within-host priority effects that give advantage to the second pathogen to infect a host increase the likelihood of pathogen coexistence by increasing the fitness of pathogens at low prevalence (figs. 4, 5). These findings integrate within-host priority effects into the modern coexistence framework of frequency dependence (Chesson 2000; Adler et al. 2007). Our study also identifies destabilizing mechanisms in previous studies where pathogens could not coinfect a single host (Gupta et al. 1994). In these systems, the first pathogen to infect a host blocked infection from any other pathogens. This blocking can be interpreted as an extreme case of within-host priority effects that benefit the first pathogen to arrive in a host, which our results indicate should strongly reduce coexistence. Given the ubiquity of within-host priority effects in naturally coinfected systems (Hoverman et al. 2013; Natsopoulou et al. 2015; Wuerthner et al. 2017), the ability to make systematic predictions about their consequences for disease dynamics is valuable. For example, malaria strains benefit from first arrival in hosts (de Roode et al. 2005), thus within-host priority effects may decrease strain diversity in malaria, decreasing host mortality and the evolution of virulence. As new and reemerging infectious diseases spread through coinfected human and wildlife populations, better understanding the consequences of within-host priority effects might allow us to better understand which new diseases will reach endemic states and which will eventually be outcompeted.

Our results also expand the understanding of priority effects and coexistence that has been established in freeliving systems. Fukami et al. (2016) point out that latearriving species in a patch will initially have a low relative abundance compared to early arriving species. If an orga- nism's relative fitness increases with its relative abundance, then this acts as a destabilizing mechanism (Chesson 2000), resulting in competitive exclusion of late-arriving species. However, the role of priority effects as a stabilizing coexistence mechanism due to second-arriver advantage has been overlooked. This is not because there are no examples of second-arriver advantage in free-living organisms (Fukami 2015). However, priority effects occur as a stabilizing mechanism when second-arriver advantage at the patch (or host) scale alters coexistence at the metacommunity (or host population) scale. Studies explicitly linking priority effects at the patch scale to coexistence at the metacommunity scale are rare and have focused on firstarriver advantage scenarios (Urban and De Meester 2009; Tucker and Fukami 2014). Given the many examples of second-arriver advantage in free-living organisms (Fukami 2015), we should consider how priority effects may act as a stabilizing coexistence mechanism across both free-living and within-host communities.

Our results provide a framework to understand when within-host priority effects should alter pathogen coexistence in natural populations. First, an invading or rare pathogen must encounter heterospecific pathogens within hosts at an appreciable rate. In our system, we find up to $35 \%$ prevalence of Pasteuria in Daphnia dentifera (M. A. Duffy, unpublished data). Thus, when rare, Metschnikowia infections have up to a 1 in 3 chance of infecting hosts previously infected by Pasteuria, thus boosting spore yield and increasing Metschnikowia invasion ability. Second, within-host priority effects will be important for determining coexistence when there are large fitness differences between pathogens (fig. 6B). This is likely in our system, as Metschnikowia and Pasteuria have different responses to environmental factors such as light and predation (Auld et al. 2014; C. L. Shaw, S. R. Hall, E. P. Overholt, C. E. Cáceres, C. E. Williamson, and M. A. Duffy, unpublished data). Third, our study demonstrates that the impact of within-host priority effects on pathogen fitness must be strong compared to the average impact of coinfection on pathogen fitness to alter coexistence. The average fitness of a pathogen is determined by (a) the proportion of infected hosts that are coinfected and $(b)$ the proportion of coinfected hosts in which the pathogen arrives first or second. For Pasteuria, fitness is determined almost entirely by $a$, which is not determined by Pasteuria prevalence (fig. 5). Thus, including within-host priority effects does not change Pasteuria fitness when rare. For Metschnikowia, fitness is determined more by the order of arrival in coinfections than the coinfection proportion (fig. 1). Whether Metschnikowia can persist in a population therefore changes when we include within-host priority effects. Adding withinhost priority effects to models of coinfected populations inevitably increases their complexity. Examining the above factors will allow us to determine when this added complexity is necessary to understand infectious disease patterns. 
Links between within-host and between-host processes create feedbacks that drive host-pathogen dynamics. Our work illustrates how between-host processes (prevalence and transmission) alter within-host dynamics via withinhost priority effects. To fully apply this framework to pathogen communities in other natural systems, we must understand how within-host priority effects function in species-rich pathogen and parasite assemblages (Budischak et al. 2016). Further, we need to study whether within-host priority effects function in a discreet or continuous manner. Our study examines the order of parasite arrival while ignoring variation in the time between infection events, as do most previous experimental studies. Future work should quantify how changing the time lag between the arrival of two pathogens influences pathogen fitness. Overall, an improved understanding of how within-host priority effects influence pathogen fitness will enable us to better predict patterns of pathogen coexistence in multipathogen communities.

\section{Acknowledgments}

We would like to thank Ben Toscano, Shannon Carter, Bénédicte Bachelot, Tom Miller, Emily Schultz, Amy Dunham, Marion Donald, Raul Costa-Pereira, David Lane, and Michael Cortez for helpful conversations about this project, as well as Katherine Hunsberger for lab assistance. This project was supported by grants from the National Science Foundation (NSF; DEB-1601353, DEB-1655626, DEB-1305836, and DEB-1748729) and by the NSF Graduate Research Fellowship Program. No human or vertebrate organisms were used in this study. No competing interests exist for this study.

\section{Literature Cited}

Abu-Raddad, L. J., P. Patnaik, and J. G. Kublin. 2006. Dual infection with HIV and malaria fuels the spread of both diseases in subSaharan Africa. Science 314:1603-1606.

Adler, P. B., J. HilleRisLambers, and J. M. Levine. 2007. A niche for neutrality. Ecology Letters 10:95-104.

Alizon, S., J. C. de Roode, and Y. Michalakis. 2013. Multiple infections and the evolution of virulence. Ecology Letters 16:556-567.

Al-Naimi, F. A., K. A. Garrett, and W. W. Bockus. 2005. Competition, facilitation, and niche differentiation in two foliar pathogens. Oecologia 143:449-457.

Auld, S. K. J. R., S. R. Hall, M. A. Duffy, A. Tessier, and C. Cáceres. 2012. Epidemiology of a Daphnia-multiparasite system and its implications for the Red Queen. PLoS ONE 7:e39564.

Auld, S. K. J. R., S. R. Hall, J. Housley Ochs, M. Sebastian, and M. A. Duffy. 2014. Predators and patterns of within-host growth can mediate both among-host competition and evolution of transmission potential of parasites. American Naturalist 184(suppl.):S77S90.
Balmer, O., and M. Tanner. 2011. Prevalence and implications of multiple-strain infections. Lancet Infectious Diseases 11:868-878.

Benjamini, Y., and Y. Hochberg. 1995. Controlling the false discovery rate: a practical and powerful approach to multiple testing. Journal of the Royal Statistical Society B 57:289-300.

Brogden, K. A., J. M. Guthmiller, and C. E. Taylor. 2005. Human polymicrobial infections. Lancet 365:253-255.

Budischak, S. A., E. P. Hoberg, A. Abrams, A. E. Jolles, and V. O. Ezenwa. 2016. Experimental insight into the process of parasite community assembly. Journal of Animal Ecology 85:1222-1233.

Chesson, P. 2000. Mechanisms of maintenance of species diversity. Annual Review of Ecology and Systematics 31:343-366.

Civitello, D. J., S. Pearsall, M. A. Duffy, and S. R. Hall. 2013. Parasite consumption and host interference can inhibit disease spread in dense populations. Ecology Letters 16:626-634.

Clay, P. A., K. Dhir, V. H. W. Rudolf, and M. A. Duffy. 2019. Data from: Within-host priority effects systematically alter pathogen coexistence. American Naturalist, Dryad Digital Repository, http:// dx.doi:10.5061/dryad.v118180.

Cox, F. E. G. 2011. Concomitant infections, parasites and immune responses. Parasitology 122:S23-S38.

de Roode, J. C., M. E. H. Helinski, M. A. Anwar, and A. F. Read. 2005. Dynamics of multiple infection and within-host competition in genetically diverse malaria infections. American Naturalist 166:531-542.

Devevey, G., T. Dang, C. J. Graves, S. Murray, and D. Brisson. 2015. First arrived takes all: inhibitory priority effects dominate competition between co-infecting Borrelia burgdorferi strains. BMC Microbiology 15:61.

Fenton, A., T. Lamb, and A. L. Graham. 2008. Optimality analysis of Th1/Th2 immune responses during microparasite-macroparasite co-infection, with epidemiological feedbacks. Parasitology 135:841853.

Fitt, B. D. L., Y.-J. Huang, F. van den Bosch, and J. S. West. 2006. Coexistence of related pathogen species on arable crops in space and time. Annual Review of Phytopathology 44:163-182.

Fukami, T. 2015. Historical contingency in community assembly: integrating niches, species pools, and priority effects. Annual Review of Ecology, Evolution, and Systematics 46:1-23.

Fukami, T., E. A. Mordecai, and A. Ostling. 2016. A framework for priority effects. Journal of Vegetation Science 27:655-657.

Gardner, A., S. A. West, and A. Buckling. 2004. Bacteriocins, spite and virulence. Proceedings of the Roval Societv B 271:1529-1535.

Goodman, R. M., and A. F. Ross. 1974. Enhancement by potato virus $\mathrm{Y}$ of potato virus $\mathrm{X}$ synthesis in doubly infected tobacco depends on the timing of invasion by the viruses. Virology 58:263271.

Gupta, S., J. Swinton, and R. M. Anderson. 1994. Theoretical studies of the effects of heterogeneity in the parasite population on the transmission dynamics of malaria. Proceedings of the Roval Societv B 256:231-238.

Halliday, F. W., J. Umbanhowar, and C. E. Mitchell. 2017. Interactions among symbionts operate across scales to influence parasite epidemics. Ecology Letters 20:1285-1294.

Hood, M. E. 2003. Dynamics of multiple infection and within-host competition by the anther-smut pathogen. American Naturalist $162: 122-133$

Hoverman, J. T., B. J. Hoye, and P. T. J. Johnson. 2013. Does timing matter? how priority effects influence the outcome of parasite interactions within hosts. Oecologia 173:1471-1480. 
Jackson, J. A., R. J. Pleass, J. Cable, J. E. Bradley, and R. C. Tinsley. 2006. Heterogeneous interspecific interactions in a host-parasite system. International Journal for Parasitology 36:1341-1349.

Jager, I., and S. Schorring. 2006. Multiple infections: relatedness and time between infections affect the establishment and growth of the cestode Schistocephalus solidus in its stickleback host. Evolution 60:616-622.

King, K. C., S. K. J. R. Auld, P. J. Wilson, J. James, and T. J. Little. 2013. The bacterial parasite Pasteuria ramosa is not killed if it fails to infect: implications for coevolution. Ecology and Evolution 3:197-203.

Lawn, S. 2004. AIDS in Africa: the impact of coinfections on the pathogenesis of HIV-1 infection. Journal of Infection 48:1-12.

Leung, T. L. F., and R. Poulin. 2011. Intra-host competition between co-infecting digeneans within a bivalve second intermediate host: dominance by priority-effect or taking advantage of others? International Journal for Parasitology 41:449-454.

Levin, B. R., and J. J. Bull. 1994. Short-sighted evolution and the virulence of pathogenic microorganisms. Trends in Microbiology 2:76-81.

Lion, S., and J. A. J. Metz. 2018. Beyond $R_{0}$ maximisation: on pathogen evolution and environmental dimensions. Trends in Ecology and Evolution 33:458-473.

Lohr, J. N., M. Yin, and J. Wolinska. 2010. Prior residency does not always pay off-co-infections in Daphnia. Parasitology 137:14931500 .

Marchetto, K. M., and A. G. Power. 2017. Coinfection timing drives host population dynamics through changes in virulence. American Naturalist 191:173-183.

May, R. M., and M. A. Nowak. 1994. Superinfection, metapopulation dynamics, and the evolution of diversity. Iournal of Theoretical Biology 170:95-114.

- 1995. Coinfection and the evolution of parasite virulence. Proceedings of the Roval Societv B 261:209-215.

Mordecai, E. A., K. Gross, and C. E. Mitchell. 2016a. Within-host niche differences and fitness trade-offs promote coexistence of plant viruses. American Naturalist 187:E13-E26.

Mordecai, E. A., A. G. Jaramillo, J. E. Ashford, R. F. Hechinger, and K. D. Lafferty. 2016b. The role of competition-colonization tradeoffs and spatial heterogeneity in promoting trematode coexistence. Ecology 97:1484-1496.

Natsopoulou, M. E., D. P. McMahon, V. Doublet, J. Bryden, and R. J. Paxton. 2015. Interspecific competition in honeybee intracellular gut parasites is asymmetric and favours the spread of an emerging infectious disease. Proceedings of the Roval Society B 282: 20141896.

Nowak, M. A., and R. M. May. 1994. Superinfection and the evolution of parasite virulence. Proceedings of the Roval Society B 255:8189.

Ojosnegros, S., N. Beerenwinkel, T. Antal, M. A. Nowak, C. Escarmís, and E. Domingo. 2010. Competition-colonization dynamics in an RNA virus. Proceedings of the National Academv of Sciences of the USA 107:2108-2112.

Overholt, E. P., S. R. Hall, C. E. Williamson, C. K. Meikle, M. A. Duffy, and C. E. Cáceres. 2012. Solar radiation decreases parasitism in Daphnia. Ecology Letters 15:47-54.

Pedersen, A. B., and A. Fenton. 2007. Emphasizing the ecology in parasite community ecology. Trends in Ecology and Evolution 22:133-139.
Petney, T. N., and R. H. Andrews. 1998. Multiparasite communities in animals and humans: frequency, structure and pathogenic significance. International Journal for Parasitology 28:377-393.

Power, A. G. 1996. Competition between viruses in a complex plantpathogen system. Ecology 77:1004-1010.

Rasmussen, N. L., B. G. Van Allen, and V. H. W. Rudolf. 2014 Linking phenological shifts to species interactions through sizemediated priority effects. Lournal of Animal Ecology 83:12061215.

Rolff, J., and M. T. Siva-Jothy. 2003. Invertebrate ecological immunology. Science 301:472-475.

Sandoval-Aguilar, J. A., A. W. Guzmán-Franco, J. K. Pell, S. J. Clark, R. Alatorre-Rosas, M. T. Santillán-Galicia, and G. ValdovinosPonce. 2015. Dynamics of competition and co-infection between Zoophthora radicans and Pandora blunckii in Plutella xylostella larvae. Fungal Ecology 17:1-9.

Shocket, M. S., A. T. Strauss, J. L. Hite, M. Šljivar, D. J. Civitello, M. A. Duffy, C. E. Cáceres, and S. R. Hall. 2018. Temperature drives epidemics in a zooplankton-fungus disease system: a traitdriven approach points to transmission via host foraging. American Naturalist 191:435-451.

Tessier, A. J., and P. Woodruff. 2002. Trading off the ability to exploit rich versus poor food quality. Ecology Letters 5:685-692.

Tucker, C. M., and T. Fukami. 2014. Environmental variability counteracts priority effects to facilitate species coexistence: evidence from nectar microbes. Proceedings of the Roval Society B 281:20132637.

Urban, M. C., and L. De Meester. 2009. Community monopolization: local adaptation enhances priority effects in an evolving metacommunity. Proceedings of the Roval Societv B 276:41294138.

Vasco, D. A., H. J. Wearing, and P. Rohani. 2007. Tracking the dynamics of pathogen interactions: modeling ecological and immune-mediated processes in a two-pathogen single-host system. Lournal of Theoretical Biology 245:9-25.

Wale, N., D. G. Sim, and A. F. Read. 2017. A nutrient mediates intraspecific competition between rodent malaria parasites in vivo. Proceedings of the Roval Society B 284:20171067.

Wuerthner, V. P., J. Hua, and J. T. Hoverman. 2017. The benefits of coinfection: trematodes alter disease outcomes associated with virus infection. Iournal of Animal Ecology 86:921-931.

\section{References Cited Only in the Online Appendixes}

Hirsch, M. W., and S. Smale. 1974. Differential equations, dynamical systems, and linear algebra. Academic Press, San Diego, CA.

Regoes, R. R., J. W. Hottinger, L. Sygnarski, and D. Ebert. 2003. The infection rate of Daphnia magna by Pasteuria ramosa conforms with the mass-action principle. Epidemiology and Infection 131:957-966.

Searle, C. L., C. L. Shaw, K. K. Hunsberger, M. Prado, and M. A. Duffy. 2016. Salinization decreases population densities of the freshwater crustacean, Daphnia dentifera. Hydrobiologia 770:165-172.

Stevens, M. H. 2009. A primer of ecology with R. Springer, New York.

Associate Editor: Greg Dwyer Editor: Daniel I. Bolnick 